\title{
TTR
}

Traduction, terminologie, re?daction

\section{Au-delà de la pensée binaire en traductologie : esquisse d'une analyse sociologique des positions traductives en traduction littéraire}

\section{Beyond Binary Thinking in Translation Studies: Towards a Sociological Analysis of Translational Positions in Literary Translation}

\author{
Jean-Marc Gouanvic
}

Volume 19, numéro 1, 1er semestre 2006

Figures du traducteur/Figures du traduire I

Figures of Translators/Figures of Translation I

URI : https://id.erudit.org/iderudit/016662ar

DOI : https://doi.org/10.7202/016662ar

Aller au sommaire du numéro

Éditeur(s)

Association canadienne de traductologie

ISSN

0835-8443 (imprimé)

1708-2188 (numérique)

Découvrir la revue

Citer cet article

Gouanvic, J.-M. (2006). Au-delà de la pensée binaire en traductologie : esquisse d'une analyse sociologique des positions traductives en traduction littéraire. TTR, 19(1), 123-134. https://doi.org/10.7202/016662ar
Résumé de l'article

En traductologie, les notions généralement admises, telles que « source » et " cible ", " propre " et " étranger ", " domestication " et " foreignization ", " adequacy " et " acceptability » ont tendance à offrir de la traduction une image binaire. Cet article tente de penser la traduction au-delà du binarisme, en particulier du couple "interne/externe ", en redéfinissant de façon succincte les notions d'habitus, de champ, de capital symbolique, d'homologie, d'illusio (Bourdieu) et de signifiance (Meschonnic) en sociologie de la traduction. La notion d'habitus est examinée à propos de Maurice-Edgar Coindreau et de Marcel Duhamel, et celle d'illusio est appliquée, conjointement avec celle de signifiance, aux traductions de The Grapes of Wrath (J. Steinbeck) par Karin de Hatker et Albert Debaty ainsi que de A Farewell to Arms (E. Hemingway) par Maurice-Edgar Coindreau. L'article conclut à l'utilité des notions héritées de Bourdieu et de Meschonnic pour penser la traduction en dehors du binarisme.
Tous droits réservés (c) TTR: traduction, terminologie, rédaction Les auteurs, 2007
Ce document est protégé par la loi sur le droit d'auteur. L'utilisation des services d'Érudit (y compris la reproduction) est assujettie à sa politique d'utilisation que vous pouvez consulter en ligne. 


\title{
Au-delà de la pensée binaire en traductologie : esquisse d'une analyse sociologique des positions traductives en traduction littéraire
}

\author{
Jean-Marc Gouanvic
}

\section{Introduction}

La traduction échappe difficilement à l'a priori qui consiste à la penser comme si c'était un donné et non pas un construit. Penser la traduction comme si c'était un donné, c'est tendre la plupart du temps à faire de la traductologie une réflexion sur ses effets et sur ses usages; c'est la considérer comme un produit, en risquant de succomber à un fonctionnalisme du pire, où peuvent régner comportements cyniques et intentions retorses ou, à l'inverse, pratiques enchantées auxquelles est déniée une analyse des conditions de production. Si la traduction est bien un construit, il s'agit de la construction d'une pratique. Mais comment analyser la pratique de la traduction, en évitant l'écueil des notions déréalisantes - et proprement scolastiques (Bourdieu)? Comment éviter les discours du sens commun sur la traduction, avec ces notions chevillées à son exercice, que sont « source » et " cible», " départ » et " arrivée », " fond » et « forme », etc.? Comment ne pas tomber dans les binarités plus sophistiquées du « propre » et de l' " étranger », ou encore de la " domestication » et " foreignization »? Il est fort possible que ces notions dissimulent, plutôt qu'elles ne révèlent, la nature de ce qui est à l'œuvre dans la traduction. Elles révèlent la surface d'un phénomène par l'emploi de notions telles que celles d' " équivalence » ou de conformité à la lettre ou au sens des textes ou des discours originaux. Ou encore elles préconisent une autre binarité théorique, celle de l'« adéquation » et de l'« acceptabilité ». En 
fait, cette révélation ou ce dévoilement est partiel et il peut jouer un rôle dans l'analyse des faits de traduction. Malheureusement, il est fondé sur des distinctions qui sont d'abord dans l'esprit de ceux qui les proposent et non pas tant dans la présentation des faits qu'ils sont censés analyser. Ces notions risquent d'être des écrans qui masquent les phénomènes et leur complexité en les simplifiant outrancièrement. Peuvent tomber sous le coup de cette critique les théories axées sur la cible, on les aura reconnues sans peine, aussi bien que celles axées sur la source.

\section{Objectivation et binarité « interne/externe » en traductologie}

Il est un type d'analyse traductologique qui établit comme naturelle (illusoirement, inutile de le dire) une disparité entre ce qui relève de l'externe et ce qui relève de l'interne, ce qui concerne la traduction comme texte et ce qui concerne son mode d'insertion dans l'institution. On est ici au cœur du problème posé au binarisme intellectuel : comment co-penser " interne/externe » (Bourdieu, 1984, p. 216) en traduction? Comment penser une traductologie qui ne reproduise pas purement et simplement les schèmes binaires traductionnels, tout en demeurant " objectivation », l'objectivation de toute connaissance scientifique? Comment rendre compte de l'objet d'étude (la traduction) sans refouler le sujet (le traductologue) hors des limites de l'entreprise objectivante? Nous avons abordé cette question ailleurs (à paraître en 2007) et nous ne pouvons qu'y renvoyer le lecteur. Nous voudrions ici nous concentrer sur certaines idées de Pierre Bourdieu situées en deçà de la question de l'objectivation et les appliquer à la traduction en analysant la pratique de la traduction en dehors de ces schèmes.

Habitus, champ, capital symbolique, illusio, toutes ces notions heuristiques construites par Pierre Bourdieu (1997) ${ }^{1}$ pour rendre raison de la pratique sociale sont au centre de sa pensée sur la littérature. Cependant, on ne peut demander à sa théorisation d'être applicable telle quelle à tous les objets, et en particulier à la pratique de la traduction. Ainsi, la traduction, bi-culturelle, ne peut s'analyser que comme une pratique mixte, métissée, hybride. Certes, les notions d'habitus et de légitimité sont ouvertes aux contacts entre langues, entre cultures, entre sociétés. Rien dans l'habitus n'établit chez Bourdieu que les

\footnotetext{
1 On peut se reporter à Méditations pascaliennes (1997), l'un des essais importants de Bourdieu, pour une introduction à ces notions clé de la pensée du sociologue.
} 
dispositions et la trajectoire sociale ne valent que pour les agents indigènes saisis dans leurs comportements purement nationaux (si d'ailleurs de tels comportements existent). Il en va de même pour la notion de légitimité, dont la généralité garantit l'application à la traduction. Par contre, les notions de champ et d'illusio n'ont que très rarement été appliquées à la traduction; or, l'enjeu d'une sociologie de la traduction réside dans l'articulation de ces notions avec la traduction. Nous examinerons également, quoique succinctement, la notion de signifiance, que nous conjoignons à celle d'illusio, pour rendre raison de la pratique de la traduction dans les textes.

\section{La traduction dans le champ littéraire}

Nous allons brièvement analyser sur des pratiques concrètes de traduction comment s'insèrent les notions d'habitus, de champ et d'illusio dans un nouveau modèle traductologique non fondé sur des paradigmes binaires. Nous emprunterons des cas de traduction à la littérature américaine dans la France des années 1930 et 1940 (Gouanvic, 2007).

Voyons d'abord la notion de champ. En traduction littéraire, la notion de champ vaut pour la littérature et non pour la traduction, et cela pour des raisons simples (elles deviendront claires dans les exemples ci-dessous) qui se résument dans un fait indéniable: les traductions trouvent leur place dans les champs littéraires à côté des œuvres indigènes sans constituer des structures autonomes et spécifiques, à part entière. Il en découle que le champ dont il sera question ici est le champ littéraire français et les traductions sont celles qui ont été injectées dans le marché français de l'édition pendant les années 1930 et 1940.

En tout premier lieu, les années 1930 et 1940 voient une recrudescence des traductions de l'américain en français. Qu'il s'agisse d'Hemingway, de Dos Passos, Faulkner, Steinbeck, les œuvres sont publiées dans des collections de traductions d'éditeurs prestigieux (comme « Du monde entier » de Gallimard ou comme « le Cabinet cosmopolite » de Stock, Delamain et Boutelleau) ou par de petites maisons d'édition telles que les Nouvelles Éditions Latines, les Belles Lettres, les Éditions du Pavois, la Jeune Parque, etc. Ces petites maisons sont parfois des antennes des maisons dominantes et les traductions qui y sont publiées sont alors transférées dans ces dernières sans difficulté pour être republiées. 
Traiter les traductions comme appartenant au champ de la littérature française permet de saisir des enjeux des traductions d'Hemingway, Dos Passos, etc., pour la littérature française, en analysant comment les traducteurs de ces auteurs américains font bouger les hiérarchies de légitimités socio-esthétiques dans le champ littéraire français. Au contact d'une littérature étrangère, la littérature française est transformée, parfois considérablement, parfois de manière infinitésimale, et les enjeux se trouvent déplacés dans le champ. C’est exactement ce qui s'est passé avec les œuvres traduites des auteurs mentionnés. Chacun dans leur ordre, ces auteurs imposent de nouvelles légitimités dans le champ littéraire français. On en a une preuve évidente dans la réception critique des uns et des autres, orchestrée entre autres par André Malraux, André Maurois, Jean-Paul Sartre, Valery Larbaud, Raymond Las Vergnas, Claude-Edmonde Magny. La belle unanimité des critiques est l'expression explicite d'une formidable légitimité de la culture américaine dans la culture française de l'entre-deux-guerres. Même Maurice-Edgar Coindreau, qui ne reconnaît pas à Hemingway, Steinbeck, etc., la stature de grands écrivains, a dans son panthéon personnel les écrivains américains William Faulkner, William Goyen, Flannery O’Connor.

La manière dont il est possible d'analyser la littérature traduite appartient-elle à la traductologie? Sans aucun doute. Mais une étude potentiellement complète d'un champ culturel national à une époque donnée est une entreprise si considérable qu'il convient que ce soit un historien qui l'effectue avec ses outils spécifiques. On en a un excellent exemple récent avec la thèse de Blaise Wilfert soutenue en 2003 intitulée Paris, la France et le reste... Importations littéraires et nationalisme culturel en France, 1885-1930. L'auteur y analyse dans le détail les relations conflictuelles entre maisons d'édition et entre traducteurs et conclut que la traduction a servi à alimenter les débats nationaux dans un sens inattendu, la nationalisation de la vie intellectuelle. Une telle étude historique du champ littéraire du point de vue de la traduction est essentielle au traductologue. Elle fait intervenir l'autre notion fondatrice de la théorie bourdieusienne, celle d'habitus. Ce que permet l'habitus, c'est d'envisager la traduction dans la perspective des agents de la traduction, de leurs dispositions et de leur trajectoire sociale. Ainsi les divers agents de la traduction trouvent tout naturellement leur place et leur position dans le modèle traductologique : directeurs de maisons d'édition, directeurs de collection, agents littéraires, écrivains traduits et, bien entendu, traductrices et traducteurs. 


\section{Habitus du traducteur et traduction ${ }^{2}$}

Comment se définit l'habitus du traducteur? Formé dans les luttes antérieures menées dans les champs, il se définit dans la relation avec les enjeux du présent et est tourné vers l'à-venir qui se dessine dans les comportements qui s'offrent à lui. Plutôt que projet, il est protention pré-réflexive vers l'à-venir ${ }^{3}$. L'habitus repose sur le socle de l'habitus primaire ou originel, composé d'un substrat de comportements hérités dans l'enfance, et est actualisé sous la forme de l'habitus spécifique. L'habitus spécifique est lui construit sur des dispositions de l'agent qui trouve à s'investir dans les champs et qui en retour modifie le champ où il a trouvé intérêt à exercer son action. Par exemple, Maurice-Edgar Coindreau, traducteur de Dos Passos, d'Hemingway et de Faulkner, est doté d'un habitus primaire marqué par ses origines vendéennes, qu'il interprète dans ses activités de traducteur sous la forme d'une détestation pour Hemingway, Steinbeck, etc., et une prédilection pour les écrivains du Sud des États-Unis, Faulkner en tout premier lieu, qu'il assimile homologiquement aux Chouans (1974). Sa trajectoire sociale de traducteur est celle de l'agrégé d'espagnol, traducteur des auteurs américains et de Dos Passos tout d'abord, rencontré en Espagne dans les années 1920. Il sera le promoteur et le traducteur, un peu malgré lui, d'Hemingway et de la littérature américaine à partir de 1928. Sa manière de traduire est celle de l'universitaire, professeur à Princeton, formé en France au contact de la littérature haute, laissant très peu de place aux vernaculaires dans les romans qu'il traduit. Par ses traductions il imprime au champ de la littérature française un modèle de comportement et de style qui contribue à le structurer et à le renforcer. On le voit aussi à propos de Marcel Duhamel (1972), qui est aux antipodes de la trajectoire sociale de Coindreau, du fait que son habitus primaire est d'une nature différente. Duhamel n'a pas continué ses études au-delà du certificat d'études primaires, mais, d'une intelligence vive, il a appris l'anglais sur le tas, petite main dans un hôtel de son oncle à Manchester pendant la Première Guerre mondiale. Son habitus est proche de celui de son ami Jacques Prévert, avec qui il a été associé pendant les premières années de l'aventure surréaliste. D’abord doubleur de cinéma, il est passé à la traduction littéraire

\footnotetext{
${ }^{2}$ Voir notre ouvrage (2007), qui aborde dans le détail l'habitus des traducteurs, en particulier celui de Maurice-Edgar Coindreau et celui de Marcel Duhamel.

${ }^{3}$ Voir tout particulièrement Méditations pascaliennes (1997) aux pages 248249.
} 
progressivement pour donner toute sa mesure à la fin de la Deuxième Guerre mondiale et après, en créant la collection de la Série Noire en 1945 et en traduisant plusieurs auteurs américains réalistes.

Quels sont les enseignements d'une traductologie informée par les idées de champ et d'habitus? Si la trajectoire sociale de l'agent traducteur est formée dans et par la partie traductive du champ littéraire, les principales déterminations de l'exercice de la profession de traductrice ou de traducteur trouvent leur origine dans le champ : prise de position, statut professionnel, etc. Champ et habitus sont deux versants d'une même réalité : l'habitus habite le champ et le champ habite l'habitus, l'un et l'autre s'étayant mutuellement. On est déjà ici aux antipodes de toute pensée binaire.

Demandons-nous maintenant comment est traitée l'opposition traditionnelle entre les pratiques externes et les pratiques internes, c'est-à-dire entre les déterminations qui concernent les champs et les habitus et celles qui regardent les pratiques internes, à savoir les textes. On sait combien la problématique textuelle est fondamentale en traductologie : c'est sur elle que repose la pratique de l'agent traducteur et c'est elle qui la détermine.

\section{Traduire l’illusio littéraire}

Traduire un texte littéraire n'est pas traduire un texte à visées fonctionnelles. Il y a quelque chose de spécifique dans le littéraire, ce que Bourdieu (1992) nomme l'illusio littéraire. Or, l'illusio littéraire est liée aux enjeux du champ littéraire qu'un lecteur, par exemple, trouve intérêt à jouer à propos d'un texte particulier. Le lecteur va s'investir dans le jeu de la lecture, en se prenant au jeu; l'illusio littéraire repose sur une pratique non consciente qui est, pour reprendre la formule de Coleridge (1907), a willing suspension of disbelief $f^{4}$. Que fait le traducteur dans ces conditions? Il est censé recréer tous les traits du texte de telle sorte que le lecteur fasse une expérience de lecture dans la langue de traduction qui soit comparable à l'expérience de lecture dans la langue originale. Nous disons bien " censé recréer ", car cela ne va pas du tout de soi. On se souvient des fameuses tendances déformantes

\footnotetext{
${ }^{4}$ Cependant, cette formule n'est pas entièrement satisfaisante. En effet, elle laisse entendre que la " suspension de la non-croyance » est délibérée (ce que suppose willing), alors qu'elle relève beaucoup plus des comportements réflexes que des comportements conscients.
} 
d'Antoine Berman, lesquelles sont intrinsèques à toute traduction. L'enjeu intériorisé par l'écrivain et par le traducteur serait potentiellement identique, d'une part si les textes littéraires étaient des réalités entièrement généralisables ou d'autre part si ces réalités étaient purement abstraites. Traduire l'illusio d'un texte littéraire se heurte à de nombreuses difficultés : l'incommensurabilité des grammaires et des lexiques dans les diverses langues, la polysémie des textes littéraires, leur genèse socio-historique dans les cultures (les champs) originels et la différence essentielle avec la culture où le texte est traduit (les agents étant eux-mêmes issus des sociétés où ont émergé les textes et des sociétés où ils sont traduits). La difficulté est quasi insurmontable du fait que les agents écrivains et traducteurs sont neuf fois sur dix issus de sociétés dont les traits identitaires émanent de luttes qui n'ont rien à voir les unes avec les autres dans les sociétés d'origine et de traduction (on le voit bien à propos de Coindreau et des Chouans).

Et pourtant la traduction existe, avec des résultats parfois remarquables. Il y a donc quelque chose qui passe de l'original en traduction, quelque chose qui est loin d'être négligeable. Nous faisons l'hypothèse que, si quelque chose passe, c'est en vertu d'une homologie essentielle (1987, p. 175) entre sociétés, cultures, textes, l'homologie étant définie par Bourdieu comme une " ressemblance dans la différence », un peu comme, en linguistique, Jakobson (1966) définit toute communication verbale comme une « équivalence dans la différence ». Mais la ressemblance/différence bourdieusienne est sociale et étrangère à tout fonctionnalisme ${ }^{5}$. Nous ne nous étendrons pas sur cet aspect de la traduction. Voyons plutôt comment l'illusio des textes originels est rendue en traduction, en y intégrant la notion de signifiance (Meschonnic, 1999).

\section{Signifiance, illusio et traduction}

La signifiance est, pour Meschonnic, liée au rythme dans le discours. Il écrit : le rythme est « l'organisation et la démarche même du sens dans le discours » (1999, p. 99). C'est «l'organisation (de la prosodie à

\footnotetext{
${ }^{5}$ Sur cette question du fonctionnalisme, voir notamment Choses dites (1987) aux pages 127-128, où Bourdieu fait la critique de la notion de " stratégie ", notion fonctionnaliste s’il en est : « [Ce terme] encourage le paralogisme fondamental, celui qui consiste à donner le modèle qui rend raison de la réalité pour constitutif de la réalité décrite, en oubliant le "tout se passe comme si", qui définit le statut propre du discours théorique. » (p. 127)
} 
l'intonation) de la subjectivité et de la spécificité d'un discours : son historicité », « la signifiance généralisée d'un discours. Ce qui s’impose immédiatement comme l'objectif de la traduction » (ibid.). Meschonnic prend l'exemple simplissime et très illustratif de la sentinelle dans Hamlet (I, 1, v. 14) qui dit : «I think I hear them » (« the rivals of my watch »), où il faudrait traduire le rythme, l'oralité de l'original, la signifiance comme l'a fait Raymond Lepoutre dans la mise en scène d'Antoine Vitez en 1983 : « Je crois, je les entends » et non « je crois que je les entends » (comme toutes les traductions qui ont précédé) (p. 110), et cela indépendamment de la régle de stylistique qui veut qu'il est possible d'omettre la conjonction that dans la phrase anglaise.

Qu'en est-il dans notre corpus? On constate d'abord que les romans de Dos Passos, Hemingway, Steinbeck, Miller... ont été traités de façons extrêmement différentes en traduction. Cependant la palme est remportée par la traduction de The Grapes of Wrath (1939), intitulée Grappes d'amertume, publiée en 1944 en Belgique occupée dans la version de Karin de Hatker et Albert Debaty. Dans cette traduction, on assiste à d'innombrables déformations du texte d'origine. Tout d'abord, toute référence au mouvement ouvrier et à Marx et Lénine est gommée et l'unité de la classe ouvrière est complètement caviardée. Ensuite, la thématisation de the land, lopin de terre d'où les paysans arrachent tant bien que mal leur subsistance, est traduit systématiquement par le pays, identitaire et national. Enfin, la signifiance ultime du roman soutenue par la référence biblique aux " raisins de la colère » du titre est détournée de ce qu'elle est dans le roman de Steinbeck. La Bible dit : " Chacun mourra pour son propre crime. Tout homme qui aura mangé les raisins verts, ses propres dents seront agacées » (Jérémie, 31, 29). Pour Steinbeck les raisins, gros de la colère des pauvres, annoncent les vendanges à venir. Cette colère des pauvres contre les banques et les maisons de finance est très atténuée par la traductrice, qui la détourne de son objet (les pratiques capitalistes) en l'indexant sur le passage biblique, alors que Steinbeck la réfère lui à la solidarité et au socialisme laïc.

Pour décrire ce roman traduit, nous avons employé le terme de «signifiance » dans une acception assez différente de celle de Meschonnic, une acception qui prend en charge l'ensemble des déterminants textuels thématiques et discursifs, par exemple la présence dans le texte américain de Marx et de Lénine. Cette dimension de la signifiance n'est pas en contradiction avec la poétique du discours de Meschonnic, mais elle élargit sa perspective en y intégrant ce qui est 
traditionnellement vu comme relevant des cultures et des positions « idéologiques ». Il nous semble en effet que les aspects culturels font bel et bien partie de la signifiance des textes.

Les déformations subies par le roman traduit sont si importantes qu'elles sont immédiatement repérables sans véritablement d'appareil théorique; Grappes d'amertume est une traduction authentique (même si elle n'a strictement rien d'éthique), et non une adaptation. La plupart des traductions des auteurs mentionnés déforment le texte de façon parfois très subtile, et une théorie de la traduction très fine est indispensable. La théorie de la signifiance d'Henri Meschonnic associée à l'illusio de Bourdieu est utile à cet égard. Le versant proprement textuel de l'illusio est dans la signifiance du discours de l'original. Nous ne citerons qu'un exemple, qui est, croyons-nous, très probant, celui de $A$ Farewell to Arms traduit par Coindreau (encore lui). Le style d'Hemingway se caractérise par une extrême simplicité, qui mime la langue orale, avec des phrases sans ponctuation, et coordonnées à l'aide de and de liaison. La prosodie de ses romans est fluide, sa prose s'efforce de produire le son juste, car l'écrivain est très sensible à la voix de ses phrases. Or, le traducteur n'a pas compris la poétique d'Hemingway, et il traduit son texte sans tenir compte de toutes ces particularités, comme si son style était plat, banalement traditionnel.

\section{Conclusion}

On voit que la théorie sociologique de Bourdieu ne suffit pas à une étude contrastive en traduction et qu'il faut y intégrer d'autres notions traductologiques. Nous pensons que la notion de " signifiance » est particulièrement adaptée à une sociologie du texte littéraire, lorsqu'elle est associée à l'illusio bourdieusienne. Le transfert d'illusio d'un texte à l'autre peut être évalué à l'aide de l'analyse de la signifiance, cette signifiance étant en quelque sorte la garante de l'homologie des illusio. À notre sens, l'avantage qu'il y a à associer illusio et signifiance réside dans la socialisation de la notion de signifiance; car l'absence de binarité est intrinsèque aux deux notions et elle est reconduite dans la notion conjointe que nous proposons en sociologie traductologique. Si la traduction est plus ou moins tendue entre deux pôles principaux (source et cible, etc.), habitus, champ, capital symbolique, légitimité, homologie, illusio, signifiance sont propres à offrir de cette pratique une analyse qui rende compte de sa complexité, au-delà des oppositions simples et de surface. C'est peut-être dans l'analyse des notions de 
légitimité, d'homologie, d'illusio et de signifiance, et notions similaires, que se trouve l'un des enjeux des recherches en sociologie de la traduction.

\section{Université Concordia}

\section{Références}

BERMAN, Antoine (1984). « Traduction ethnocentrique et traduction hypertextuelle ». L'Écrit du temps 7, pp. 109-123.

- (1985). « La traduction comme épreuve de l'étranger ». Texte 4, pp. 67-81.

BOURDIEU, Pierre (1984). Questions de sociologie. Paris, Minuit.

- (1987). Choses dites. Paris, Minuit.

- (1992). Les Règles de l'art. Genèse et structure du champ littéraire. Paris, Seuil, coll. « Libre examen ».

—(1997). Méditations pascaliennes. Paris, Seuil, coll. « Liber ».

COINDREAU, Maurice-Edgar (1974). Mémoires d'un traducteur. Paris, Gallimard.

COLERIDGE, Samuel Taylor (1907). Biographia Literaria. Ed. with his Aesthetical Essays, by J. Shawcross, 2 vol. Oxford, The Clarendon Press.

DUHAMEL, Marcel (1972). Raconte pas ta vie. Paris, Mercure de France.

GOUANVIC, Jean-Marc (2007). Pratique sociale de la traduction. Le roman réaliste américain dans le champ littéraire français (19201960). Arras, Artois Presses Université, coll. « Traductologie ».

- (à paraître). « Objectivation, réflexivité et traduction : Pour une relecture bourdieusienne de la traduction ». Dans Translation Sociologya new discipline under construction. Michaela Wolf et Alexandra Fukari, dir. Université de Graz (Autriche). 
HEMINGWAY, Ernest (1931). L'Adieu aux armes (A Farewell to Arms). Trad. de Maurice-Edgar Coindreau. Préface de Drieu La Rochelle. Paris, NRF, coll. « Du monde entier ».

JAKOBSON, Roman (1966 [1959]). " On Linguistic Aspects of Translation ». On Translation, Reuben A. Brower, dir. Oxford, Oxford University Press, pp. 232-239.

MESCHONNIC, Henri (1999). Poétique du traduire. Lagrasse, Verdier. Voir en particulier « Rythme et traduction », pp. 97-111.

STEINBECK, John (1944). Grappes d'amertume (The Grapes of Wrath). Trad. de Karin de Hatker, texte définitif d'Albert Debaty. Bruxelles, Les éditions De Kogge, coll. « Messages ».

TOURY, Gideon (1995). Descriptive Translation Studies and Beyond. Amsterdam/Philadelphia, John Benjamins, coll. " Benjamins Translation Library ».

WILFERT, Blaise (2003). Paris, la France et le reste... Importations littéraires et nationalisme culturel en France, 1885-1930. Thèse de doctorat. Université Paris I Panthéon - Sorbonne, UFR d’histoire.

RÉSUMÉ : Au-delà de la pensée binaire en traductologie : esquisse d'une analyse sociologique des positions traductives en traduction littéraire - En traductologie, les notions généralement admises, telles que « source » et « cible », " propre » et " étranger », « domestication » et " foreignization », " adequacy » et " acceptability » ont tendance à offrir de la traduction une image binaire. Cet article tente de penser la traduction au-delà du binarisme, en particulier du couple « interne/externe », en redéfinissant de façon succincte les notions d'habitus, de champ, de capital symbolique, d'homologie, d'illusio (Bourdieu) et de signifiance (Meschonnic) en sociologie de la traduction. La notion d'habitus est examinée à propos de MauriceEdgar Coindreau et de Marcel Duhamel, et celle d'illusio est appliquée, conjointement avec celle de signifiance, aux traductions de The Grapes of Wrath (J. Steinbeck) par Karin de Hatker et Albert Debaty ainsi que de A Farewell to Arms (E. Hemingway) par Maurice-Edgar Coindreau. L'article conclut à l'utilité des notions héritées de Bourdieu et de Meschonnic pour penser la traduction en dehors du binarisme. 
ABSTRACT: Beyond Binary Thinking in Translation Studies: Towards a Sociological Analysis of Translational Positions in Literary Translation - In translation studies, generally admitted notions such as "source" and "target», the "self» and the « foreign », « domestication » and « foreignization », " adequacy » and «acceptability ", tend to offer a binary image of translation. This article attempts to think translation beyond binarisms, particularly the « internal/external » dichotomies, by briefly defining the notions of habitus, field, symbolic capital, homology, illusio (Bourdieu) and signifiance (Meschonnic) in sociology of translation. The notion of habitus is examined à propos Maurice-Edgar Coindreau and Marcel Duhamel and illusio is applied, along with signifiance, re-interpreted in sociology of translation, to the translations of The Grapes of Wrath (J. Steinbeck) by Karin de Hatker and Albert Debaty and of A Farewell to Arms (E. Hemingway) by Maurice-Edgar Coindreau. In conclusion, the article highlights the usefulness of notions drawn from Bourdieu and Meschonnic in order to apprehend translation beyond surface binarisms.

Mots-clés : sociologie de la traduction, habitus, champ, illusio, homologie.

Keywords: sociology of translation, habitus, field, illusio, homology.

Jean-Marc Gouanvic: Département d'Études françaises, Université Concordia, Bureau LB-631-10, 1455, boul. de Maisonneuve Ouest, Montréal (Québec), H3G 1 M8.

Courriel : jmgouan@alcor.concordia.ca 